\title{
Fast PET Preview Image Reconstruction, Streaming, and Visualization During Data Acquisition: A Preliminary Study
}

Xiaoying Ji, Yang-Ming Zhu, Bin Zhang, and Andriy Andreyev

Advanced Molecular Imaging, Philips HealthTech, Cleveland, Ohio

\begin{abstract}
PET acquisition and reconstruction are time-consuming. A PET preview image is commonly reconstructed at the end of data acquisition of each bed-position frame in the step-and-shoot mode. We propose a scheme to reconstruct, stream, and visualize the PET preview image during acquisition to provide quasi-real-time visual feedback. Methods: As acquisition proceeds, event data are processed continuously by a backprojection method using time-of-flight kernels while corrections are applied only for sensitivity, time span, and decay. A preview update can be scheduled by frame or by a configured time interval. To create a preview image, the 3-dimensional volume of the current segment is knit with other existing segments. The knitted volume is projected onto a 2-dimensional plane, and the resultant gray-scale image is streamed to a display component for visualization. Results: By using fast and simple reconstruction and correction, the described scheme balances processing speed and image quality to provide early and frequent visual feedback. Results show that the preview creation, streaming, and visualization time are shorter than the acquisition time for a typical whole-body study. Conclusion: Fast feedback is achieved during PET acquisition, which provides clinicians with an indication of data acquisition and an estimation of image quality and allows early corrective measure and image quality control if necessary.
\end{abstract}

Key Words: PET preview; quality control; time of flight

J Nucl Med Technol 2019; 47:243-248

DOI: 10.2967/jnmt.118.218511

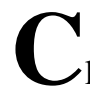

linicians, particularly technologists, are always looking for fast and accurate feedback during PET scanning. A preview image can serve that purpose. It is desirable that such feedback be provided in real time or quasi-real time and as early and frequently as possible. However, unlike other modalities such as ultrasound or CT, commercial PET/CT products do not support real-time or quasi-realtime preview image generation and display during PET acquisition. Preview images are either updated after each bed-position acquisition is finished or at the end of the

Received Jul. 24, 2018; revision accepted Dec. 24, 2018.

For correspondence or reprints contact: Yang-Ming Zhu, Advanced

Molecular Imaging, Philips HealthTech, 595 Miner Rd., Cleveland, OH 44143.

E-mail: ymzhu@ieee.org

Published online Feb. 15, 2019

COPYRIGHT (c) 2019 by the Society of Nuclear Medicine and Molecular Imaging. complete acquisition, with a delay on the order of one to a couple of minutes. Clinicians check PET preview images and release the patient if they confirm that the data acquisition has been performed as planned and the image is of the desired quality; otherwise, the acquisition is adjusted and the patient is rescanned.

A full data acquisition usually takes from 1 to $3 \mathrm{~min}$ per frame (1). A frame-by-frame preview update during data acquisition will result in 1-3 min (plus an additional delay to create the preview image) with no image information, let alone if the preview image is generated after the entire data acquisition completes. Vendors often provide count-rate information in real time, but preview images are much appreciated for a quick and visual inspection. The approach implemented in commercial PET/CT products not only provides little feedback to clinicians during acquisition but also limits efficiency.

Any incorrect acquisition setup may not be detected until the first preview image is generated. In that case, time is wasted because there is no opportunity for the clinicians to correct the errors sooner. If the patient needs to be rescanned after a long, bad scan is discarded, the additional scan reduces the effective scanner use, increases the waiting time of other patients already scheduled, and disrupts the normal clinical workflow and patient scheduling. Moreover, reinjection of PET tracers and a CT rescan may need to be prescribed if rescanning is required, which increases cost and radiation exposure to the patient and clinicians. Because of the radiotracers' decay effects, the increased waiting time for other patients who have already been administered the radiotracer may lead to a reduction in image quality or an increased data acquisition time, which may further disrupt the normal clinical workflow.

Considering these potential issues related to a long preview-image generation time, we propose a scheme to create, stream, and visualize a fast preview image in order to shorten the wait for the preview and provide acquisition feedback to clinicians with a configurable updating interval in seconds or by frame. The proposed scheme consumes minimal system resources so that the normal data processing and reconstruction are not adversely affected. The images are for preview purposes only, and diagnostic-quality images are still reconstructed with full-featured reconstruction algorithms. 


\section{MATERIALS AND METHODS}

Our proposed scheme has 3 main components as illustrated in Figure 1: data acquisition, PET preview module, and display application.

\section{Data Acquisition}

Data acquisition (the left part of Fig. 1) collects PET event data and streams the data to the PET preview module (the middle part of Fig. 1). No other significant modification to the existing acquisition module is required except the data streaming requirement. In our prototype simulation, list-mode files of raw PET event data are served as input to the PET preview module reconstruction program. The data were acquired on a Vereos Digital PET/CT scanner (Philips). In practice, list-mode data will be collected continuously and transferred to the PET preview module based on a configured time interval (e.g., 1, 3, or $5 \mathrm{~s}$ ) or at the end of each frame.

\section{Preview Display}

Besides common functions supported in a PET/CT product, the console in our proposed scheme displays the preview image annotated with statistical data collected during acquisition by the preview module. The display application is a client to the PET preview module. It connects to the PET preview module at startup. As the PET preview image is being reconstructed, the display application keeps receiving incoming packets sent by the preview module and displays the preview images and statistics as quasi-real-time feedback.

A sample display used in our experiment is shown in Figure 2. It has a connection setup (Internet protocol address and port number), an image display area, a textbox for progression information and statistical data, and a configuration part for projection methods (maximum or sum intensity projection) to be applied to the volumetric data to generate a 2-dimensional (2D) preview image.

\section{PET Preview Module}

As the key component in the scheme, the PET preview module computes and converts PET list-mode data into a PET preview image. The module takes reconstruction parameters from configuration files, which specify what data will be collected, how the data will be processed, and how often the preview will be generated and sent to the display console. Typical configuration parameters that users set up for fast feedback include projection method, updating time interval, and image size, among others. On the basis of the image quality requirement on preview images, clinicians can adjust some configuration parameters on the console display to achieve interactive communication with the PET preview module, if desirable.

Meanwhile, certain statistics can be collected and computed from data embedded in the event data stream, such as number of prompt events or cardiac beating statistics. The collected statistical data are sent to the console display application together with

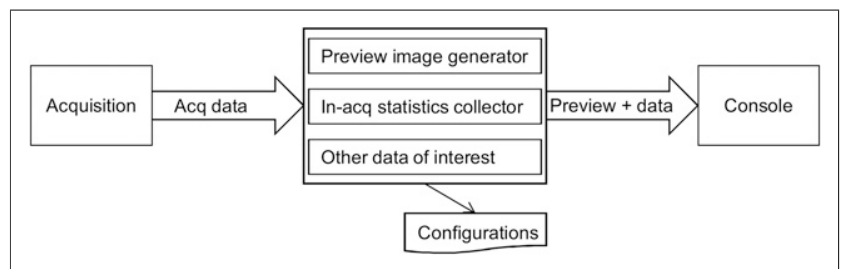

FIGURE 1. Software components and data flow in quasi-realtime image streaming scheme. the preview image. The details of preview generation are further explained below.

\section{Reconstruction}

Full-fledged reconstruction involves massive computation, which is not feasible for fast PET preview image generation (2,3). Fast visual feedback during acquisition requires balance between processing speed and image quality. We choose to apply a backprojection (2) method with time-of-flight (TOF) capability $(1,4-6)$ to provide a good-quality preview and quick reconstruction at high frequency, with limited resource use so that the computing resources can be used for data acquisition and high-quality image reconstruction.

With 2 crystal positions and corresponding TOF information, the position of the annihilation event can be located on the line of response with up to a TOF resolution interval. The position of the annihilation event can be determined in 2 different ways (4). The first option, known as the most likely position, maps the location on the basis of the measured TOF difference, assuming a perfect timing resolution. The second option uses a gaussian-shaped TOF kernel around the most likely position, and the timing resolution is used to control the width of the gaussian kernel. The image quality and processing time of the 2 alternatives are different and will be investigated. Backprojections are performed event by event, as the events are streamed from data acquisition.

\section{Corrections}

Considering the purpose of preview and processing speed, we applied 3 fundamental corrections: a geometric sensitivity correction, a decay correction, and a time-span correction. Further corrections can be introduced depending on the computational demand and image quality needs. For example, if a CT volume is available, attenuation correction can be applied, which improves the attenuation of the events coming from deep inside the patient or going through strong-attenuation tissues such as bones. However, for timely service of the preview purpose, simple corrections concerning the processing speed should be considered.

Geometric Sensitivity Correction. The probability that an annihilation will be detected by detection crystals is related to the position of the event with respect to the scanner geometry. In general, along the axial direction, the scanner sensitivity is decreasing from the center to the edges. We apply geometric sensitivity correction to remedy this effect. The sensitivity matrix is calculated (e.g., by a Monte Carlo simulation (7) or by doing a backprojection of all feasible lines of response) and saved in advance, so that during reconstruction of the preview image, the sensitivity parameter for each voxel can be read directly without computation.

Decay and Time-Span Correction. We apply decay correction each time the image data are knit. The radioactive decay function is described as follows:

$$
A(t)=A_{0} e^{-\lambda t}, \lambda=\frac{\ln 2}{T},
$$

where $A$ is the isotope activity, $T$ is the half-life time, $t$ is the decay time, and $A_{0}$ is the initial activity at time $t=0$. For a time interval $\left[t_{x}, t_{x}+\Delta t\right]$, where $t_{x}$ is the bed-position acquisition start time and $\Delta t$ is the bed-position acquisition time span, the average amount of radioactive decay can be calculated by (8).

$$
\hat{A}=A_{0} \frac{1}{\lambda \Delta t} e^{-\lambda t_{x}}\left(1-e^{-\lambda \Delta t}\right)
$$

This parameter is calculated and used to correct each set of image data. 


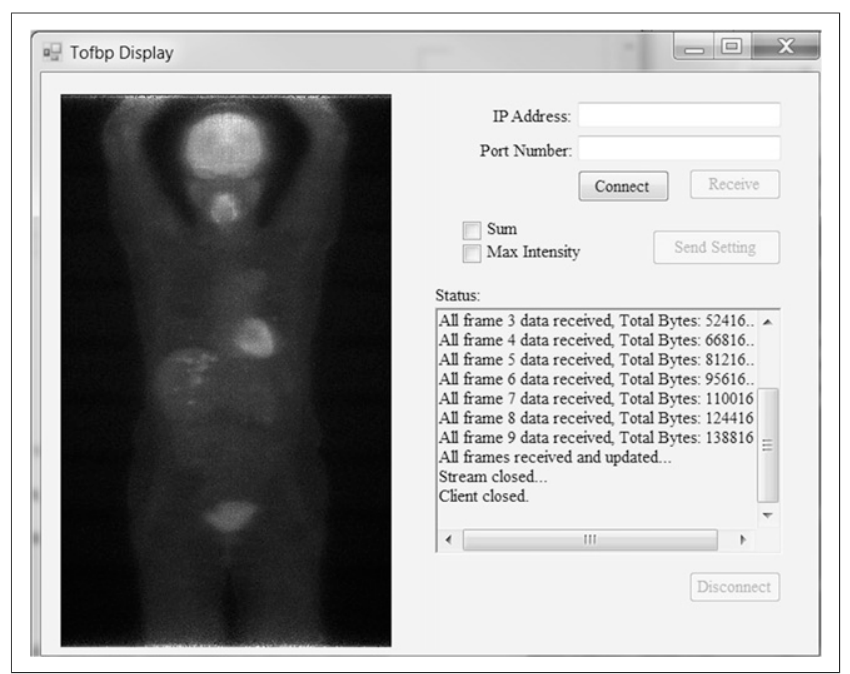

FIGURE 2. Sample display program.

The number of data updated and displayed for the most recent frame might be less than for the previous frames, such as when the program is streaming the first $2 \mathrm{~s}$ of data for frame 4 and all data for the first 3 frames have already been streamed. Because the image data are cumulative, fewer data may lead to dark areas in the displayed image, which is not informative for clinicians. Therefore, we further applied time-span correction so that the number of data is normalized for each frame displayed. For the $n$ seconds of data displayed for the newest frame in a data acquisition of $m$ seconds per frame, the $n$ seconds of data are multiplied by $m / n$. Because no frame time information is available for the first frame, the time-span correction is skipped for the first frame. Because noise is amplified when events for a very short time are streamed for preview, the timespan correction may introduce notable window width or level changes during the in-frame update. To deal with the unexpected sudden change, window width or level is changed only after completion of image creation for each frame, except for the first frame.

\section{Data Knitting and Maximum-Intensity Projection}

Image data are knit when an update is needed, namely, at the end of data acquisition for each bed position or every $n$ seconds of data collected. The image data are first multiplied by the correction parameters discussed above, and then the overlapping slices between 2 frames are knit together using weights given by a sigmoid function. Although other schemes to choose the weights are possible and used in practice, the sigmoid function allows one to control the smoothness of the weights.

Suppose there are $a$ overlapping slices between 2 frames, which are numbered from 0 to $a-1$ from edge to center in both frames. Then, for slice $i$ the weight $w$ is

$$
w(i)=\frac{1}{1+e^{-x\left(i-\frac{a-1}{2}\right)}},
$$

where $x$ is a parameter that controls the slope of the function. It can be adjusted to achieve the best image quality. The slice in the overlap region is composed of the weighted sum of the 2 slices. For example, when there are 16 slices overlapped and $x=0.45$, the weight is shown in Figure 3.

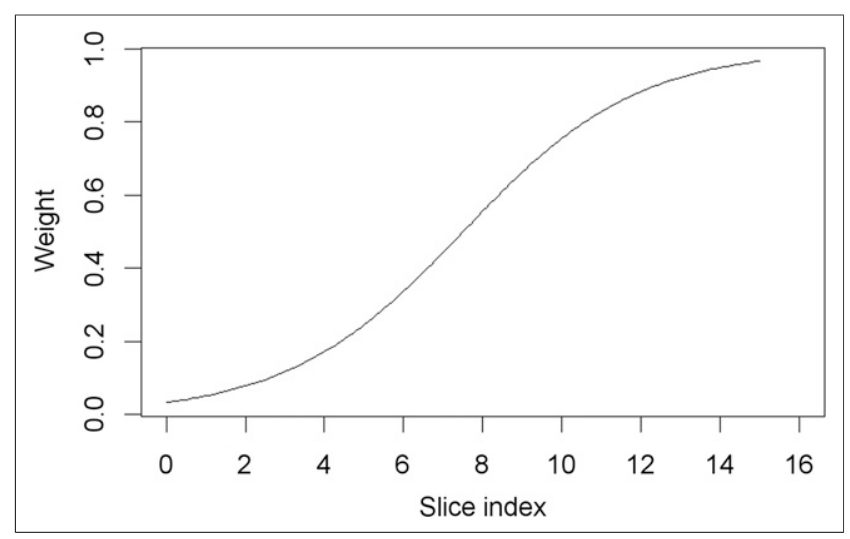

FIGURE 3. Sigmoid function used to calculate weights when knitting images.

Knitted 3-dimensional (3D) image data are projected onto a 2D coronal plane with the maximum-intensity projection, which selects the maximum value of all voxels along a projection ray, with possible depth cueing. When a sum projection is used, all voxel values along the projection ray are simply accumulated instead.

The 2D image data are then converted into a gray-scale image with intensity $0-255$, which is sent to the display client. The display application reads the gray-scale data and displays them properly. Configurations for display-related parameters can be applied during this process to adjust the image representation.

\section{Patient Data and Experiment Setup}

We simulated and implemented fast streaming with wholebody patient data and phantom data, but here we show data for only 1 patient for simplicity and because of space limitations. To respect patient privacy, the only patient demographic available is body weight $(45.8 \mathrm{~kg})$. The institutional review board approved this retrospective study, and the requirement to obtain informed consent was waived. The isotope was ${ }^{18} \mathrm{~F}$, with a half-life of around $109.8 \mathrm{~min}$. The nominal TOF timing resolution was 320 ps. The injection amount was $505.79 \mathrm{MBq}$, and the scan started at around $59 \mathrm{~min}$ after injection. The input list file included 9 frames, with $90 \mathrm{~s}$ of data per frame. 3D PET images were reconstructed using TOF-based backprojection. Corrections were applied for sensitivity, time span, and decay. The 3D images had a voxel size of $2 \times 2 \times 2 \mathrm{~mm}$. A larger voxel size reduces computational time at the expense of degraded image quality. The 3D PET image data were then projected onto a 2D coronal plane with maximum-intensity projection, which was the image used for preview purposes. The full $2 \mathrm{D}$ preview image was 288 pixels in width and 482 pixels in length, with each frame being 288 pixels in width and 82 pixels in length. Different backprojection methods and configured updating intervals were applied and analyzed, whereas image quality and processing time were visually assessed. The single threaded preview generation was done on a personal computer with 8-GB random access memory and an Intel Core $2.70-\mathrm{GHz}$ i7-4800MQ central processing unit. The constraint of single threaded implementation was purposely put in place so that when the utility is deployed in a clinical environment, it will not compete with other components for computing resources. On a clinical system, most computing 


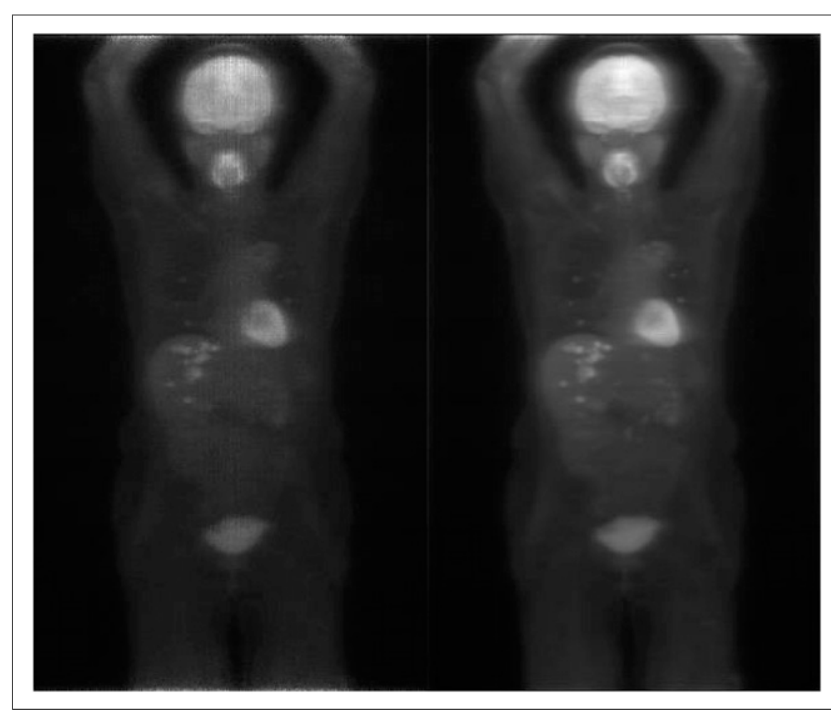

FIGURE 4. Example preview images: TOF-center backprojection (left) and full backprojection (right).

resources are allocated to time-consuming reconstruction and other system management tasks.

\section{RESULTS}

\section{Backprojection}

An annihilation event can either be distributed with probability weighting (gaussian-shaped TOF kernel) by ray tracing (full backprojection method) or be directly located at the most likely position (center-of-TOF-kernel backprojection method) on a line of response. The computation for the TOF-center backprojection method is more efficient than ray tracing at the cost of image quality.

According to our implementation, the computation of the TOF-center backprojection method with a frame-by-frame update takes around $125 \mathrm{~s}$ for 9 frames, or $14 \mathrm{~s}$ per frame. The computation of the full backprojection method with a frame-by-frame update takes around $650 \mathrm{~s}$, or $72 \mathrm{~s}$ per frame. Example imaging results are shown in Figure 4. In the figure, the preview image computed by the full backprojection method has more detail, but the computing time was close to the data acquisition time. Meanwhile, the TOF-center backprojection method took much less time than the acquisition time, allowing additional corrections to be applied as long as the processing time was within the data acquisition time. Any transmission delay during data transfer is considered, and processing time and image quality are eventually balanced. Philips application specialists, most of whom were technologists by training, gave overwhelmingly positive feedback on the clinical utility of the scheme and on the preview image quality and speed performance.

\section{Fast Preview Updating}

Figure 5 shows the results when the preview image was streamed and updated frame by frame.

Figure 6 shows the in-frame update when the preview image was updated with every $2 \mathrm{~s}$ of data. A 1-s update interval was also attempted with success. As the data acquisition time for frame 4 increased, noise decreased, and the quality of the preview was notably increased. All these figures were generated using the TOF-center backprojection method.

The frame-based update scheme (Fig. 5) is not available on all clinical scanners. The in-frame update (Fig. 6) is not supported on any scanner at this point. Both Figure 5 and Figure 6 indicate the data acquisition progression and provide visual feedback, which increases user experience with the system. Although the preview image is not intended for clinical diagnosis, clinicians can explicitly recognize whether the acquisition setup is right or whether the acquisition should be interrupted for correction. Diagnostic-quality images are reconstructed using the full-fledged reconstruction methods provided on scanners.

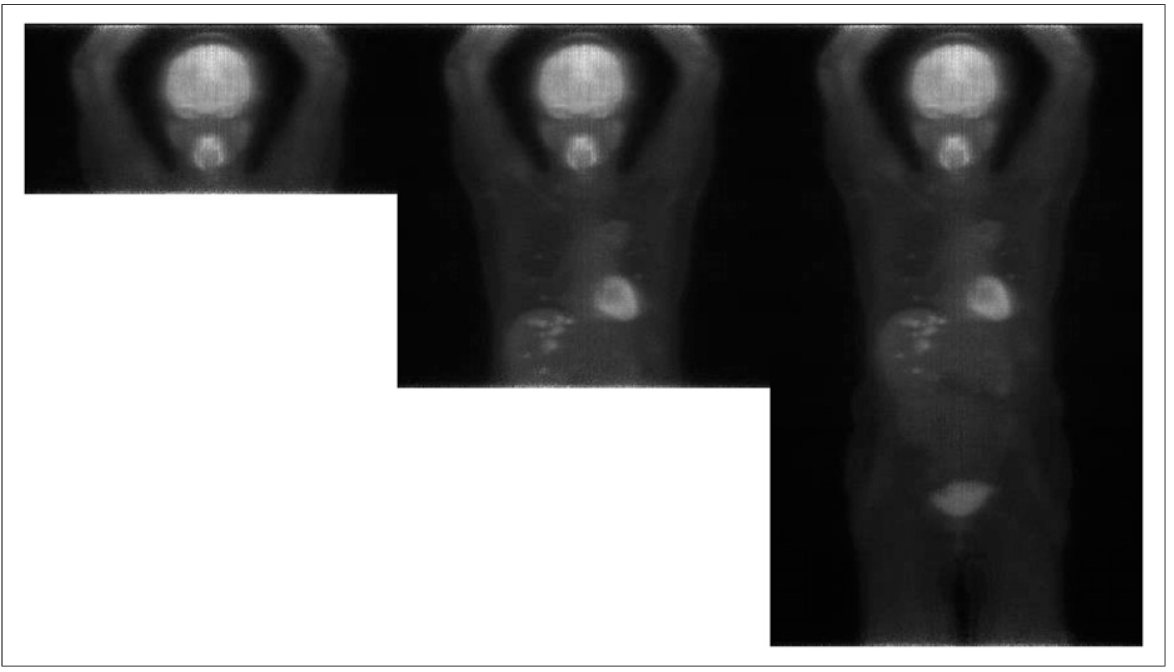

FIGURE 5. From left to right, preview images at end of second, fifth, and ninth frames.

\section{DISCUSSION}

The proposed streaming aims for a fast and frequent preview update, which can be used for multiple purposes. The PET preview image alone can demonstrate the progression of data acquisition, which improves the user experience with the system, as shown in Figures 5 and Figures 6. Simple setup mistakes such as blank images or incorrect positioning of the patient in the field of view can be detected and corrected as early as possible so that the wasted time is minimized, thus controlling interruptions and disruptions to the 


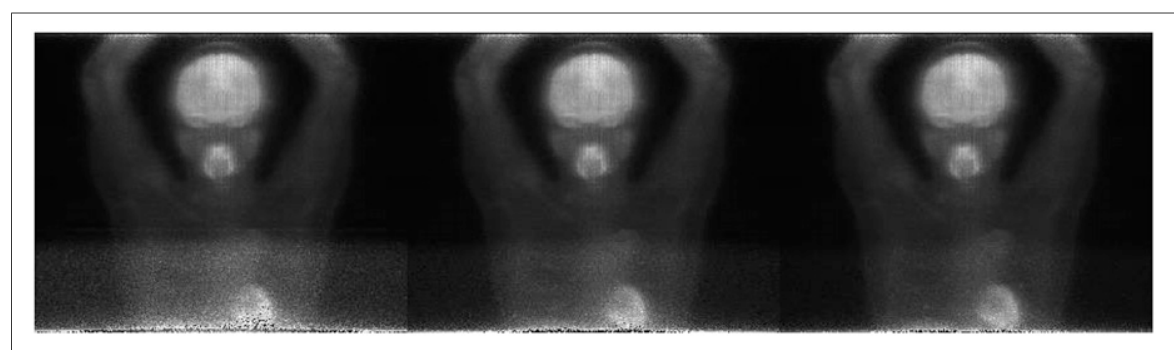

FIGURE 6. From left to right, preview images for fourth frame with 4, 10, and $20 \mathrm{~s}$ of data.

workflow and reducing the risk of misdiagnosis, rescanning, and an additional dose prescription. In modern hybrid scanners such as PET/CT, these simple mistakes can be avoided during the planning stage. However, the PET component can still be used as a standalone device. Even if it is used as a hybrid device, some vendors offer linked or unlinked planning options, and the simple mistakes might happen with the unlinked planning. In consideration of image quality, the feedback provides an estimate about the data acquisition and the full-fledged reconstructed image. The dynamic communication between clinicians and the preview creation module provides the possibility to adjust data acquisition parameters on the fly to meet clinical objectives (e.g., to increase or decrease the acquisition time for a bedposition frame). The clinical utility of the proposed method can be further explored to see whether it can detect unexpected circumstances such as patient motion during data acquisition. One may expect that the magnitude of motion and the motion pattern may determine whether motion can be detected reliably and how soon. To answer this and related questions, a systematic experimental study is necessary in which one can exploit a phantom setup with deliberately introduced motion.

The proposed method carefully trades off the processing speed and image quality. Because the method processes event-by-event based directly on the list-mode data, the processing time naturally depends on the activity. Large activities or higher count rates have a longer processing time. The concern is then whether the processing can catch up with the event stream. As the patient example shows, for a typical whole-body oncologic study, the processing time is $14 \mathrm{~s}$ which is far less than the bed-position acquisition time (typically $90 \mathrm{~s}$ ). Thus, the current approach can accommodate activities a few times larger than that of the typical whole-body oncologic study. For a cardiac study, the activities might be much higher. If the processing cannot catch up with the event stream, dropping events at random could be an option. On the basis of the portion of events dropped, the reconstructed images can optionally be scaled to compensate for the drop. We do not expect the processing time to strongly depend on the acquisition volume, since the event is put directly on the most likely position (there is no expensive ray tracing). Many factors affect image quality, including scanner hardware, processing software and algorithms, study protocols, and patient demographics. For example, patients with a higher body mass tend to have a lower image quality, if everything else is the same. A systematic study is needed to assess whether the current tradeoff decisions are valid under a variety of conditions. Modifications might be required under different conditions.

Besides direct display, the PET preview images may also be fused with CT images (volumetric image or surview) if available. The fusion can be performed either on the display application or during preview image creation. If the images are fused on the display application, clinicians can adjust fusion parameters, such as $\alpha$-blending transparency; however, fusion on the display application also introduces extra computational burden on the display. If the images are fused during the preview reconstruction, then colored image are streamed across the process or machine boundary, increasing the amount of data transferred and potentially causing a transmission delay.

The updating time interval may be customized by application requirements or configured by clinicians. In the roughest case, the preview image can be updated frame-by-frame (Fig. 5). In the most frequent case, the preview image can be updated second-by-second (Fig. 6). A fixed time interval can be applied in the continuous-couch-motion acquisition mode. A varying updating time interval may be implemented as well. For instance, for fast indication of the correctness of data acquisition, the first frame may be updated more frequently. As acquisition proceeds, the updating speed may be slowed to reduce computation time and network transmission delay.

The proposed approach is easy to implement according to our experience in our development context and has minimal impact on the existing software system. Some adaptation may be necessary when our approach is attempted in a different environment. However, we do not encourage users to implement it themselves without first consulting the scanner vendor, since such implementations may violate the warranty or certifications.

\section{CONCLUSION}

In commercial PET/CT products, PET preview is provided at the end of each frame acquisition or the complete acquisition. This paper has presented a scheme that provides an instant and frequently updated visual feedback to clinicians. To reduce computational burden, the TOF-center backprojection algorithm and simple corrections, including sensitivity correction, time-span correction, and decay correction, are proposed and applied in this work. Even though the preview image is not intended for clinical diagnosis, the preview provides feedback on the data acquisition progression and allows for early corrective measures if anything is 
not as expected during acquisition. Further investigation may be performed on how to balance image quality and processing time so that the significance of the preview image can be further improved. This work may also be extended to non-TOF data with sophisticated methods in image generation-for example, a fast maximum-likelihood expectation maximization algorithm for each update or other efficient alternatives - provided that the preview generation will not consume too much computing resources.

\section{DISCLOSURE}

All authors were Philips employees when the work was conducted. No other potential conflict of interest relevant to this article was reported.

\section{ACKNOWLEDGMENTS}

The patient data are courtesy of Drs. Michael V. Knopp and Jun Zhang and were acquired at Ohio State University using a precommercial investigational Philips Vereos Digital
PET/CT device. We are grateful to Sydney Kaplan, who proofread the manuscript and improved its presentation.

\section{REFERENCES}

1. Vandenberghe S, Mikhaylova E, D'Hoe E, Mollet P, Karp JS. Recent developments in time-of-flight PET. EJNMMI Phys. 2016;3:3.

2. Sciascio E, Manni A, Guzzardi R. Design of an architecture for real-time 3D PET imaging. Real-Time Imaging. 1998;4:255-262.

3. Cui JY, Pratx G, Prevrhal S, Levin C. Fully 3D list-mode time-of-flight PET image reconstruction on GPUs using CUDA. Med Phys. 2011;38: 6775-6786.

4. Snyder D, Thomas L, Ter-Pogossian M. A mathematical model for positronemission tomography systems having time-of-flight measurements. IEEE Trans Nucl Sci. 1981;28:3575-3583.

5. Karp JS, Surti S, Daube-Witherspoon M, Muehllehner G. Benefit of time-of-flight in PET: experimental and clinical results. J Nucl Med. 2008;49:462-470.

6. Conti M. Why is TOF PET reconstruction a more robust method in the presence of inconsistent data? Phys Med Biol. 2011;56:155-168.

7. Thompson CJ, Moreno-Cantu J, Picard Y. PETSIM: Monte Carlo simulation of all sensitivity and resolution parameters of cylindrical positron imaging systems. Phys Med Biol. 1992;37:731-749.

8. NEMA NU 2-2012: Performance Measurements of Positron Emission Tomographs (PETs). Rosslyn, VA: National Electrical Manufacturers Association; 2013.

\section{Erratum}

In the article "Protocols for Harmonized Quantification and Noise Reduction in Low-Dose Oncologic ${ }^{18}$ F-FDG PET/CT Imaging," by Machado et al. (J Nucl Med Technol. 2019;47:47-54), the value 1.00 was inadvertently left out of the OSEM3D column (first row) in Table 1 during copyediting. The corrected table (with missing value italicized) appears below. We regret the error.

TABLE 1

HBIs for Groups of Acquisition Parameters and Different Reconstruction Settings

\begin{tabular}{|c|c|c|c|c|c|}
\hline Acquisition parameter & OSEM3D & PSF7 & PSF2-EQ6 & PSF2-EQ6.5 & PSF2-EQ7 \\
\hline Group A: 1,272 MBq·s/kg & 1.00 & 1.02 & 1.13 & 1.08 & 1.13 \\
\hline Group B: $416 \mathrm{MBq} \cdot \mathrm{s} / \mathrm{kg}$ & 1.00 & 1.00 & 1.17 & 1.02 & 1.06 \\
\hline Group C: $216 \mathrm{MBq} \cdot \mathrm{s} / \mathrm{kg}$ & 1.00 & 1.01 & 1.20 & 1.06 & 1.12 \\
\hline Group D: $81 \mathrm{MBq} \cdot \mathrm{s} / \mathrm{kg}$ & 1.29 & 1.02 & 1.40 & 1.18 & 1.15 \\
\hline
\end{tabular}

